\title{
RESPUESTA VEGETAL DE Acacia decurrens A LA INOCULACIÓN CON RIZOBACTERIAS PROMOTORAS DE CRECIMIENTOVEGETAL BAJO ESTRÉS SALINO
}

\section{VEGETAL RESPONSE OF Acacia decurrens TO INOCULATION WITH PLANT GROWTH PROMOTING RHIZOBACTERIA UNDER SALT STRESS}

\author{
Diana B. Sánchez y y Ruth R. Bonilla \\ Recibido para publicación: Enero 3 de 2014 - Aceptado para publicación: Junio 22 de 2014
}

\begin{abstract}
RESUMEN
La salinización del suelo se considera como uno de los factores ambientales limitantes del crecimiento y productividad de los cultivos en el mundo, principalmente en regiones áridas y semiáridas. Una alternativa para aliviar el estrés causado por este factor abiótico es el uso de Rizobacterias Promotoras de Crecimiento Vegetal (RPCV), las cuales pueden contribuir en el crecimiento de las plantas a través de sus múltiples funciones. El objetivo de la investigación fue realizar el aislamiento, caracterización y determinación del efecto de bacterias de vida libre halotolerantes asociadas a Acacia decurrens. Los microorganismos se caracterizaron bioquímicamente a partir de la producción de amonio, determinación de la actividad de celulosa, producción de exopolisacáridos, producción de Índoles totales, determinación cuantitativa de solubilización de fósforo y genéticamente con el 16S rRNA. Bajo condiciones de invernadero se evaluó el efecto de los microorganismos seleccionados sobre la promoción de crecimiento de Acacia decurrens a partir de variables morfométricas. Los resultados demostraron que los nueve aislamientos en estudio presentaron en general una buena actividad metabólica excepto los aislamientos ACSA12 y ACSA19 que no expresaron producción celulolítica. La respuesta de la planta evidenció que los aislamientos T4, T5 y T7 incrementaron de manera significativa la biomasa y desarrollo de la misma $(\mathrm{p} \leq 0.05)$. Los aislamientos fueron identificados genéticamente como Pantoea deleyi ACSA12, Enterobacter amnigenus ACSA14 y Serratia liquefaciens ACSA19.
\end{abstract}

Palabras clave: Enterobacter amnigenus, salinidad, Serratia Pantoea deleyi, Serratia liquefaciens.

\begin{abstract}
Soil salinization is considered as an environmental limiting factor for plant growth and crops productivity in the world, mainly in arid and semiarid regions. An alternative for alleviating the caused stress by this abiotic factor is the use of Plant Growth Promoting Rhizobacteria (PGPR). These bacteria are able to improve plant growth through its various functions. The objective of this research was the isolation, characterization and

\footnotetext{
${ }^{1 *}$ Máster en Ciencias Biológicas, Investigador Máster, C.I. Turipaná, Corporación Colombiana de Investigación Agropecuaria - CORPOICA. Km 13 vía Montería - Cereté (Colombia),Teléfono +57(1) 7691057 Ext 2249. Autor de correspondencia: dbsanchez@corpoica.org.co. ${ }^{2} \mathrm{Ph}$.D en Ciencias Agrícolas, Investigador Ph.D, Laboratorio de Microbiología de Suelos, Centro de Biotecnología e Industria, Centro de Investigación Tibaitatá. Corporación Colombiana de Investigación Agropecuaria - Corpoica. Mosquera (Colombia).
} 
effect determination of free living-halotolerant bacteria associated to Acacia decurrens. Microorganisms were biochemically characterized since ammonia production, cellulose production, exopolysaccharides production, total production of indoles and quantitative phosphorus solubilization determination and molecularly through $16 \mathrm{~S}$ rRNA. Under greenhouse conditions, it was evaluated the effect of selected microorganisms under plant growth promotion in Acacia decurrens as of morphometric variables. Results demonstrated that the nine isolates under study exhibit in general a good metabolic activity, except isolates ACSA12 and ACSA19 which did not present cellulolytic production. Plant response showed evidence that isolates T4, T5 and T7 increased significantly biomass and plant development. These isolates were genetically identified as Pantoea deleyi ACSA12, Enterobacter amnigenus ACSA14 y Serratia liquefaciens ACSA19.

Key words: Enterobacter amnigenus, salinity, Serratia liquefaciens, Pantoea deleyi.

\section{INTRODUCCIÓN}

La salinización del suelo se considera como un factor ambiental limitante del crecimiento y productividad de los cultivos en todo el mundo, principalmente en regiones áridas y semiáridas (Lamz y González 2013). Aunque las sales que generalmente son comunes y necesarias como componentes del suelo, en exceso pueden causar desbalances osmóticos y desequilibrio de nutrientes (Parida y Das 2005), afectando el crecimiento de las plantas, la capacidad fotosintética, la síntesis de proteínas, síntesis de lípidos, metabolitos y el contenido total del nitrógeno (Ramírez et al. 2014). En suelos del altiplano Cundiboyacense, la Acacia decurrens puede considerarse como una opción para establecer sistemas silvopastoriles en clima frío del trópico, esta especie muestra una buena adaptación a las condiciones edafoclimáticas de la zona, presenta buena tasa de crecimiento, alta producción de leña y biomasa comestible de buena calidad (Martínez y Navia 2012; Muñoz et al. 2013).

Los ecosistemas semiáridos del altiplano cundiboyacense constituyen un tipo de bioma azonal particular de Colombia, una isla biogeográfica denominada como Orobioma azonal andino del altiplano cundiboyacense; todos ellos en grave riesgo de extinción por destrucción generalizada del hábitat (Calvachi 2012). El uso de la Acacia con fines multipropósito en estos sistemas busca mejorar el nivel alimenticioy productivo de los animales, respondiendo en parte a los problemas de la deforestación, degradación del ecosistema y se proyecta como una alternativa válida para intensificar la producción y la sostenibilidad de los sistemas tradicionales (Martínez y Navia 2011). Sin embargo es indispensable mejorar su crecimiento bajo condiciones de estrés salino, característica que se está generalizando por el uso de prácticas no adecuadas como labranza y riego entre otras.

Una alternativa es el uso de Rizobacterias Promotoras de Crecimiento Vegetal (RPCV), las cuales pueden mejorar el crecimiento de las plantas bajo condiciones de estrés salino. Las bacterias promotoras de crecimiento vegetal (BPCV) Bacillus sp., Rhizobium sp., Pseudomonas sp., Azospirillum sp., entre otros géneros (Bacilio et al. 2004; Tank y Saraf 2010), toleran altas concentraciones de salinidad $y$ colonizan raíces, tallos y hojas de las plantas, permiten mejorar su crecimiento (Kang et al. 2014). Esta investigación tuvo como objetivo el aislamiento, caracterización y respuesta de la Acacia decurrens a la inoculación con bacterias de vida libre halotolerantes asociadas a esta leguminosa. 


\section{MATERIALES Y MÉTODOS}

\section{Muestreo}

El estudio se realizó en 2014, las muestras de suelo rizosférico se obtuvieron en el Centro de Investigación Tibaitatá - Corpoica ubicado en Mosquera (Cundinamarca - Colombia) a una altura de $2543 \mathrm{msnm}$; localizadogeográficamente a $4^{\circ} 41^{\prime} 43^{\prime \prime}$ de latitud norte y $74^{\circ} 12^{\prime} 30^{\prime \prime}$ de latitud oeste. Este suelo presenta una textura franco arcillosa y una conductividad eléctrica de 0,447 $\mathrm{dSm}^{-1}$. Se tomaron 3 muestras deformadas aleatoria a profundidad de $20 \mathrm{~cm}$ donde se encontraba sembrada Acacia decurrens.

\section{Aislamientos en Agar nutritivo suplementado con $0,5 \mathrm{M}$ de $\mathrm{NaCl}$}

Se pesaron $10 \mathrm{~g}$ de suelo por muestra y se diluyeron en $90 \mathrm{~mL}$ de $\mathrm{NaCl}$ estéril al 0,85\%, con agitación de 150 rpm durante 15 min. Se realizaron diluciones seriadas desde $10^{-1}$ hasta $10^{-7}$ y se inocularon $0,1 \mathrm{~mL}$ en el medio de cultivo nutritivo suplementado con 0,5 $\mathrm{M}$ de $\mathrm{NaCl}$.

\section{Efecto de la concentración de $\mathrm{NaCl}$ sobre el crecimiento bacteriano}

Se sembraron los aislamientos de bacterias Gram negativas y Gram positivas provenientes de la rizósfera de la leguminosa Acacia decurrens en agar nutritivo a diferentes concentraciones de $\mathrm{NaCl}$ 0,85 M; 1,712 M; 2,578 M; 3,424 M.

\section{Producción de $\mathrm{NH}_{3}$}

La producción de amoníaco se determinó de acuerdo a Chaney y Marbach (1962). Se inocularon los cultivos en caldo nutritivo $\left(\mathrm{gL}^{-1}\right)$ 0,5 extracto de levadura; 0,5 peptona; 0,5 caseína; 0,5 glucosa; 0,5 almidón; 0,30 $\mathrm{K}_{2} \mathrm{HPO}_{4}$ y $0,30 \mathrm{MgSO}_{4} ; \mathrm{pH}: 7,2$ por un período de $24 \mathrm{~h}$ a $30{ }^{\circ} \mathrm{C}$. Posteriormente, $1 \mathrm{~mL}$ de cada cultivo se centrifugó a 14.800 rpm durante $10 \mathrm{~min}$, se tomaron $700 \mu \mathrm{L}$ del sobrenadante $y$ fue adicionado Fenol/Nitroprusiato y Hidróxido/ hipoclorito en relación 1:1, se dejó reaccionar por 30 min a $60^{\circ} \mathrm{C}$ y se realizó la lectura a una absorbancia $630 \mathrm{~nm}$.

\section{Determinación de la actividad de celulosa}

La evaluación cualitativa de la degradación de celulosa se realizó de acuerdo con Percival et al. (2006); utilizando como medio de cultivo (carboximetilcelulosa (CMC) (gL-1): 0,2\% $\mathrm{NaNO}_{3}$; $0,1 \% \mathrm{~K}_{2} \mathrm{HPO}_{4} ; 0,05 \% \mathrm{MgSO}_{4} ; 0,05 \%$ de $\mathrm{KCl}$; $0,2 \%$ carboximetil celulosa). Se sembraron $50 \mu \mathrm{L}$ de suspensión bacteriana en solución de $\mathrm{NaCl}$ al $0,85 \%\left(\mathrm{OD}_{600}=0,500\right)$ en placa y posteriormente se incubó durante $48 \mathrm{~h}$ a $30 \pm 2{ }^{\circ} \mathrm{C}$. Transcurrido ese período, se determinó el índice de degradación (ID) coloreando las placas con rojo congo $1 \%$ y $\mathrm{HCL} 0,1 \mathrm{M}$ como revelador de los halos. Esta metodología se utilizó para las nueve cepas bacterianas seleccionadas por triplicado.

\section{Producción de Exopolisacáridos}

La determinación cuantitativa de la producción de exopolisacáridos se realizó de acuerdo con Dubois et al. (1956). Se tomó un $1 \mathrm{~mL}$ de cultivo bacteriano previamente crecido y se adicionaron $40 \mu \mathrm{L}$ de EDTA $(0,26 \mathrm{M})$ y $20 \mu \mathrm{L}$ (5M). La mezcla se centrifugó a 14.800 rpm por 10 min, se tomó el sobrenadante y se adicionó $1 \mathrm{~mL}$ de etanol helado. Posteriormente se centrifugó durante 10 min a 14.800 rpm, se eliminó el sobrenadante para re-suspender el pellet en agua destilada. Se tomaron $0,29 \mu \mathrm{L}$ y se mezclaron con fenol $5 \%$ y ácido sulfúrico concentrado. Posteriormente se estabilizó la reacción durante 10 min en baño termostatado a $30{ }^{\circ} \mathrm{C}$. La lectura se realizó a una absorbancia de $490 \mathrm{~nm}$. 


\section{Producción de Índoles totales}

Los compuestos indólicos se determinaron mediante el ensayo colorimétrico descrito por Glickmann y Dessaux (1995). Se empleó el medio de cultivo K-lactato suplementado con triptófano a $100 \mathrm{mgL}^{-1}$. Los cultivos se incubaron durante 72 h a 150 rpm en oscuridad. Para la lectura se utilizó el reactivo de Salkowsky $\left(\mathrm{FeCl}_{37} \mathrm{H}_{2} \mathrm{O}\right.$ $12 \mathrm{gL}^{-1}$ en $\mathrm{H}_{2} \mathrm{SO}_{4} 79 \mathrm{M}$ ) en una relación 1:1 con la suspensión bacteriana, dejándose reaccionar durante $30 \mathrm{~min}$ en oscuridad. Se realizó la lectura a una absorbancia de $450 \mathrm{~nm}$.

\section{Determinación cuantitativa de solubilización de fósforo}

La determinación cuantitativa de la solubilización de fósforo se realizó en medio de cultivo líquido SRS con un tiempo de incubación de 5 días a $30 \pm 2{ }^{\circ} \mathrm{C}$ a $150 \mathrm{rpm}$. Los sobrenadantes a partir del cultivo se centrifugaron a 10.000 rpm durante 10 minutos. El fosfato soluble en el sobrenadante fue estimado por el método del azul de fosfomolibdato (Fiske y Subbarow 1925).

\section{Evaluación bajo condiciones de Invernadero}

En el experimento se empleó un diseño completamente al azar con nueve tratamientos y cinco repeticiones por tratamiento en materas con una capacidad de 400 g de suelo, cada tratamiento fue inoculado con $1 \mathrm{~mL}$ de una suspensión celular con una concentración de $1 \times 10^{8}$ UFC $\mathrm{mL}^{-1}$ en medio nutritivo. Los tratamientos fueron los siguientes:

- T1: testigo no salino

- T2: testigo salino $40 \mathrm{mM} \mathrm{NaCl}$

- T3: ACSA4

- T4: ACSA12

- T5: ACSA14

- T6: ACSA17

- T7: ACSA19
- T8: ACSA21

- T9: ACSA26

- T10: ACSA27

- T11: ACSA32

La fertilización química se realizó según el análisis químico del suelo (12,23\% $\mathrm{MO} ; 2,7 \mathrm{P}$ $\left(\mathrm{mg} \mathrm{kg}^{-1}\right) ; 0,72 \mathrm{~K} ; 1,20 \mathrm{Mg}^{2}$ 2,32 Ca $\left(\mathrm{cmol}_{\mathrm{c}} \mathrm{kg}^{-1}\right)$; $\mathrm{pH}=5,5)$. La conductividad final del suelo para todos los tratamientos excepto para T1-testigo no salino fue de 0,910 dSm${ }^{-1}$. El experimento tuvo una duración de 20 días. Las variables evaluadas fueron: Longitud de la parte área $(\mathrm{cm})$, longitud radical $(\mathrm{cm})$, masa fresca de la parte aérea $(\mathrm{g})$, masa fresca de la raíz (g), masa seca de la parte aérea (g) masa seca de la raíz (g) y área foliar $\left(\mathrm{cm}^{2}\right)$. El área foliar se determinó con plantas de cada tratamiento. Estas plantas fueron luego escaneadas, obteniéndose el área foliar mediante la utilización de un medidor de área foliar $\mathrm{Cl}$ 202 Portable Leaf Area Meter (Bio-Science).

\section{Análisis estadístico}

Los datos se sometieron a evaluación estadística mediante análisis de varianza (ANOVA) y prueba de Tukey y Dunnet con 95\% de confianza.

\section{Identificación molecular de aislamientos}

Las tres cepas promisorias con mayor promoción de crecimiento de Acacia decurrens bajo condiciones de estrés por salinidad, durante el experimento en invernadero, se identificaron genéticamente usando el Kit DNA easy Blood \& Tissue (Qiagen, Alemania) siguiendo las instrucciones del fabricante. Para ello, se emplearon los iniciadores universales para la subunidad ribosomal 16S rRNA: 1492R (5' TAC GGY TAC CTT GTT ACG ACT T 3') y 27F (5' AGA GTT TGA TCM TGG CTC AG 3'). Para la PCR, se empleó el Kit PCR SuperMix (Invitrogen, Brasil) siguiendo las instrucciones del fabricante. 
Las condiciones de la PCR consistieron en una fase de denaturación inicial a $95^{\circ} \mathrm{C}$ por $5 \mathrm{~min}$, 35 ciclos de $95{ }^{\circ} \mathrm{C}$ por $45 \mathrm{~s}, 55^{\circ} \mathrm{C}$ por $45 \mathrm{~s}$ y $72{ }^{\circ} \mathrm{C}$ por 1,5 min y una extensión final a $72{ }^{\circ} \mathrm{C}$ por $10 \mathrm{~min}$. La electroforesis fue realizada en un gel de agarosa de concentración 1,2\%, empleando buffer TAE 1X y tensión de $100 \mathrm{~V}$ durante $40 \mathrm{~min}$. Para la tinción del gel, se empleó post teñido en una piscina conteniendo bromuro de etidio a una concentración de 0,5 $\mathrm{\mu gmL}^{-1}$. Los productos de PCR fueron secuenciados por la empresa CORPOGEN empleando los mismos iniciadores. Mediante la herramienta bioinformática Geneious 4.8 y la base de datos GenBank del NCBI, se extrajeron mediante BLAST las secuencias cercanamente relacionadas. Se tuvieron en cuenta los aciertos que cumplieran con un $98 \%$ de similitud y un $100 \%$ de cobertura entre la secuencia problema y las anotadas en GenBank.

\section{RESULTADOS Y DISCUSIÓN}

\section{Aislamiento y caracterización de microorganismos} provenientes de la rizósfera

De las muestras compuestas de suelo rizosférico se obtuvieron cuarenta y cuatro aislamientos Gram negativos y Gram positivos, de los cuales nueve crecieron en agar nutritivo suplementado con 2,578 $\mathrm{M}$ de $\mathrm{NaCl}$.

\section{Producción de $\mathrm{NH}_{3}$}

Todas las cepas mostraron la capacidad de producir amoníaco, principalmente las cepas ACSA26 $\left(5,97 \mu \mathrm{gmL}^{-1}\right), \operatorname{ACSA} 19\left(5,42 \mu \mathrm{gmL}^{-1}\right)$ y $\operatorname{ACSA} 14\left(3,10 \mu \mathrm{gmL}^{-1}\right)(\mathrm{p} \leq 0.05)$ (Tabla 1$)$. La producción de amoníaco es un atributo importante de las PGPR que influye en el crecimiento de las plantas indirectamente (Wani et al. 2007). Estos resultados están acordes con los reportados por Joseph et al. (2007), donde se reporta que todas las cepas de bacterias de los géneros Bacillus, Pseudomonas, Rhizobium, y Azotobacter produjeron amoníaco.

\section{Determinación de la actividad de celulosa}

Las cepas ACSA32 (5,51 mm) y ACSA26 $(4,1 \mathrm{~mm})$ presentaron los mayores índices de degradación a las $48 \mathrm{~h}$ de incubación $(\mathrm{p} \leq 0.05)$ (Tabla 1). La actividad celulolítica, además

Tabla 1. Caracterización de la promoción de crecimiento in vitro de las cepas bacterianas.

\begin{tabular}{|c|c|c|c|}
\hline Cepas & $\begin{array}{c}\text { Producción de Amonio } \\
\left(\mu \mathrm{gmL}^{-1}\right)\end{array}$ & Degradación Celulosa (mm) & $\begin{array}{c}\text { Producción de Exopolisacáridos } \\
\qquad\left(\mathrm{mgL}^{-1}\right)\end{array}$ \\
\hline ACSA4 & $2,69 \pm 0,02^{b}$ & $2,65 \pm 0,10^{\mathrm{e}}$ & $66,32 \pm 2,43^{b}$ \\
\hline ACSA12 & $2,44 \pm 0,20^{\text {bc }}$ & $2,89 \pm 0,05^{d}$ & $22,69 \pm 0,65^{\mathrm{e}}$ \\
\hline ACSA14 & $3,10 \pm 0,18^{b}$ & $0,00 \pm 0,00^{\mathrm{e}}$ & $26,90 \pm 0,22^{\mathrm{d}}$ \\
\hline ACSA17 & $1,02 \pm 0,02^{\mathrm{c}}$ & $2,98 \pm 0,02^{d}$ & $8,95 \pm 0,57^{\mathrm{gh}}$ \\
\hline ACSA19 & $5,42 \pm 0,29^{a}$ & $0,00 \pm 0,00^{\mathrm{e}}$ & $12,95 \pm 0,77^{f}$ \\
\hline ACSA21 & $0,93 \pm 0,05^{c}$ & $2,96 \pm 0,02^{\mathrm{d}}$ & $11,87 \pm 1,14^{\mathrm{fg}}$ \\
\hline ACSA26 & $5,97 \pm 0,01^{a}$ & $4,19 \pm 0,21^{b}$ & $7,22 \pm 0,61^{\mathrm{h}}$ \\
\hline ACSA27 & $2,85 \pm 0,14^{b}$ & $3,66 \pm 0,36^{c}$ & $31,32 \pm 0,31^{c}$ \\
\hline ACSA32 & $2,85 \pm 0,12^{b}$ & $5,51 \pm 0,10^{a}$ & $74,77 \pm 1,41^{a}$ \\
\hline
\end{tabular}

Medias con letras no comunes dentro de cada variable difieren estadísticamente según prueba de Tukey para $p \leq 0.05$ ( $n=3$ ). 
de su papel ecológico en la degradación de material orgánicos puede también jugar un papel importante en la supresión de enfermedades mediante la inhibición del crecimiento fúngico (Sindhu y Dadarwal 2001) a partir de la degradación de la pared celular (Mitchell y Alexander 1963). De igual forma Sindhu y Dadarwal (2001) reportan la actividad celulolítica de Pseudomonas sp. aisladas de la rizósfera de garbanzo (Cicer arietinum L.) y frijol mungo (Vigna radiata L.). Kavamura et al. (2013), obtuvieron halos con diámetros de $10 \mathrm{~mm}$ hasta $14 \mathrm{~mm}$ de degradación de carboximetil celulosa (CMC) por Bacillus sp.

\section{Producción de Exopolisacáridos}

Los resultados revelaron que todas las cepas en estudio tienen la capacidad de producir exopolisacáridos presentado los mejores resultados las cepas ACSA32 (74,77 $\left.\mathrm{mgL}^{-1}\right)$ y ACSA4 (66,32 $\left.\mathrm{mgL}^{-1}\right) \quad(\mathrm{p} \leq 0.05) \quad($ Tabla 1$)$. Estos metabolitos producidos por las células bacterianas forman una vaina órgano-mineral alrededor de las colonias, creando así un micro entorno que disminuye la pérdida de agua de las células y ayuda a su supervivencia
(Sandhya et al. 2010). Upadhyay et al. (2011) reportaron que cepas aisladas del cultivo de trigo pertenecientes a los géneros Bacillus, Burkholderia, Enterobacter, Microbacterium y Paenibacillus, produjeron exopolisacáridos, los cuales pueden ayudar a mitigar el estrés por salinidad, reduciendo el contenido de $\mathrm{Na}^{+}$ disponible para la absorción por la planta.

\section{Producción de Índoles totales}

En relación con los compuestos indólicos se observó que todas las cepas fueron capaces de producir índoles totales, presentando los mejores resultados las cepas ACSA12 $\left(98,26 \mu \mathrm{gmL}^{-1}\right)$ y ACSA4 $\left(95,57 \mathrm{\mu gmL}^{-1}\right)$ $(\mathrm{p} \leq 0.05)$ (Tabla 2). Uno de los mecanismos directos más frecuentes en la promoción de crecimiento vegetal por las bacterias es la producción de fitohormonas, como auxinas (Mohamed y Gomaa 2012). Este mecanismo es considerado uno de los más importantes, donde la auxina puede funcionar como una molécula de señalización en la regulación del desarrollo de la misma, influyendo en muchos procesos celulares como el alargamiento de las células en la planta (Ghosh et al. 2008).

Tabla 2. Caracterización de la promoción de crecimiento in vitro de las cepas bacterianas.

\begin{tabular}{ccc}
\hline Cepas & Producción de AIA $\left(\boldsymbol{\mu g m L ^ { - 1 } )}\right.$ & Fósforo Disponible $\left(\mathbf{m g L}^{-1}\right)$ \\
\hline ACSA4 & $95,57 \pm 3,01^{\mathrm{a}}$ & $99,82 \pm 1,57^{\mathrm{a}}$ \\
ACSA12 & $98,26 \pm 1,83^{\mathrm{a}}$ & $57,62 \pm 8,53^{\mathrm{b}}$ \\
ACSA14 & $60,62 \pm 1,89^{\mathrm{ab}}$ & $30,02 \pm 1,22^{\mathrm{c}}$ \\
ACSA17 & $3,30 \pm 0,23^{\mathrm{cd}}$ & $88,77 \pm 5,84^{\mathrm{a}}$ \\
ACSA19 & $58,94 \pm 1,59^{\mathrm{ab}}$ & $65,31 \pm 5,25^{\mathrm{b}}$ \\
ACSA21 & $4,48 \pm 0,18^{\mathrm{d}}$ & $100,03 \pm 2,81^{\mathrm{a}}$ \\
ACSA26 & $55,04 \pm 2,59^{\mathrm{abc}}$ & $98,09 \pm 5,31^{\mathrm{a}}$ \\
ACSA27 & $10,12 \pm 1,55^{\mathrm{d}}$ & $23,20 \pm 1,86^{\mathrm{c}}$ \\
ACSA32 & $6,65 \pm 0,85^{\mathrm{d}}$ & $26,303 \pm 4,00^{\mathrm{c}}$ \\
\hline
\end{tabular}

Medias con letras no comunes dentro de cada variable difieren estadísticamente según prueba de Tukey para $p \leq 0.05$ ( $n=3$ ). 
Costa etal. (2013) donde reportó que el 79 de sus aislados del género Bacillus, Bradyrhizobium, Rhizobium y Paenibacillus produjeron mayor cantidad de ácido 3-indolacético (AIA), cuando el medio fue suplementado con triptófano y promovieron el crecimiento del frijol caupí, esta producción de AIA se ha evidenciado que influye significativamente sobre el crecimiento y desarrollo de las plantas (Sánchez et al. 2012).

\section{Determinación cuantitativa de solubilización de fósforo}

Las cepas ACSA $21\left(100,03 \mathrm{mgL}^{-1}\right)$, ACSA4 $\left(99,82 \mathrm{mgL}^{-1}\right), \quad$ ACSA26 $\left(98,09 \mathrm{mgL}^{-1}\right) \quad y$ ACSA17 (88,77 $\left.\mathrm{mgL}^{-1}\right)$ presentaron los mayores valores $(p \leq 0.05) \quad($ Tabla 2$)$. Varios autores reportan la capacidad de solubilización de fosfato por géneros bacterianos: Azotobacter, Pseudomonas, Bacillus, Burkholderia, Erwinia, Flavobacterium, Microbacterium y Serratia (Oliveira et al. 2009; Bhattacharyya y Jha 2012; Babana et al. 2013). La producción de los ácidos orgánicos de bajo peso molecular por las rizobacterias es uno de los mecanismos más conocidos de solubilización de fosfato en el suelo (Zaidi et al. 2009); esta capacidad de aumentar la disponibilidad de fósforo se debe no sólo a la acidificación del medio en la rizósfera de las plantas, sino también de formar complejos estables de Al y Fe; estos ácidos orgánicos incrementan la disponibilidad de nutrientes, al disminuir el pH del suelo en la rizósfera (Paredes y Espinosa 2010). Sin embargo, los ácidos orgánicos no se consideran como el único factor responsable de la solubilización de fosfato por bacterias (Chen et al. 2006). Los protones asociados con los polisacáridos extracelulares secretados por los microorganismos son también responsables de la disolución de fosfato en los medios de cultivo (Illmer y Schineer 1995). Walpola y Yoon (2013) reportaron la capacidad de solubilización de fosfato inorgánico en medio líquido NBRIP de las cepas Pantoea agglomerans, Burkholderia anthina y Enterobacter ludwigii desde 624 hasta $667 \mathrm{gmL}^{-1}$.

\section{Evaluación de la respuesta vegetal de Acacia decurrens bajo condiciones de Invernadero Altura de la planta y longitud radical}

En la altura de la planta no hubo diferencias significativas $(p \leq 0.05)$ respecto al testigo salino $40 \mathrm{mM}$ de $\mathrm{NaCl}$. Sin embargo, los tratamientos T4 y T5 presentaron un incremento respecto al testigo de $17 \%$ y $16 \%$, respectivamente (Figura 1). En la longitud radical el parámetro de la tendencia fue similar, se presentó un aumento en los tratamientos T11, T5, T9 y T8 con valores de 49\%, 24\%, 19\% y 13\%, respectivamente (Figura 1). Este incremento porcentual en la variable altura de la planta y longitud raíz, puede atribuirse a la actividad de los microorganismos en la regulación fisiológica de las mismas al producir fithormonas de tipo auxinas y citoquininas, ya que estas permiten el desarrollo radicular o por ende un posible aumento en la disponibilidad de nutrientes en el suelo. Estas capacidades de las bacterias pueden promover el crecimiento de manera directa o indirecta o sinérgicamente (Joseph et al. 2007; Yasmm et al. 2007). Zahir et al. (2009) reportaron un aumento en la variable altura de la planta por encima del $21 \%$ por Serratia proteamaculans sobre el control salino ( $5 \mathrm{dS} \mathrm{m}^{-1}$ ) en plantas de trigo. Goswami et al. (2014) reportaron la promoción de crecimiento de plantas de Maní (Arachis hypogaea) con Bacillus licheniformis, simulando suelos con una concentración de 


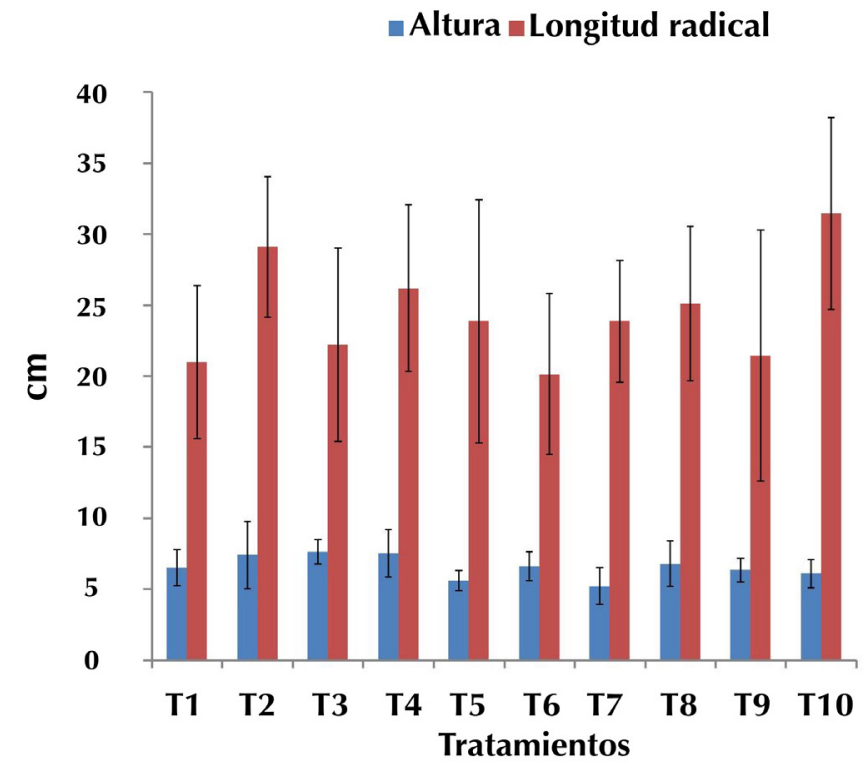

Figura 1. Altura y longitud radicular de las plantas de Acacia decurrens bajo condiciones de estrés $40 \mathrm{mM} \mathrm{NaCl}$. Cada valor es la media de cinco repeticiones. Las barras de error representan la desviación estándar. Los tratamientos son: T1 (testigo salino); T2(testigo no salino 40mM NaCl); T3(Cepa ACSA4); T4(ACSA12); T5(ACSA14); T6(ACSA17); T7(ACSA19); T8(ACSA21); T9(ACSA26); T10(ACSA27); T11(ACSA32).

$50 \mathrm{mM} \mathrm{NaCl}$ donde mostraron un aumento de altura de planta y longitud de raíz de $24 \%$ y $17 \%$ respectivamente, respecto al control no salino.

\section{Peso seco de la parte aérea y raíz}

Los resultados del peso seco de la parte aérea no evidenciaron diferencias significativas $(p \leq$ 0.05) respecto al testigo salino $40 \mathrm{mM} \mathrm{NaCl}$, sin embargo los tratamientos T4, T5 y T7 presentaron aumentos respecto a la variable de $266 \%$, 233\% y $116 \%$ respectivamente (Figura 2). Con respecto al peso seco de la raíz igualmente no se presentaron diferencias significativas $(p \leq 0.05)$ respecto al testigo, sin embargo los tratamientos T4, T3, T5 y T10 presentaron un aumento respecto al testigo salino de $232 \% \quad 160 \%$ 136\% y $72 \%$ respectivamente (Figura 2). Aún así, sin ser valores estadísticamente significativos, se evidencia un aumento porcentual que duplica la variable respuesta una o dos veces en comparación con el testigo no salino. Esta respuesta se relaciona directamente con los efectos de las bacterias en el crecimiento de la planta. Zahir et al. (2009) encontraron que una cepa de Serratia sp. produjo un aumento en la longitud y peso radicular en plantas de trigo bajo condiciones de estrés salino. Estimaron que el peso seco de la raíz aumentó en un $100 \%$ y el aéreo en un $90 \%$ con respecto a las plantas sin inocular.

\section{Área foliar}

La variable de área foliar presentó diferencias significativas $(p \leq 0.05)$ respecto al testigo salino $40 \mathrm{mM} \mathrm{NaCl}$, siendo el mejor tratamiento T7 con un incremento de $136 \%$ (Figura 3). Sin embargo T9, T3 y T5 no presentaron diferencias significativas $(p \leq 0.05)$ respecto al testigo salino $40 \mathrm{mM} \mathrm{NaCl}$; pero se obtuvieron incrementos de $81 \%$, 70\%, 67\%, respectivamente. La disminución del crecimiento observado en muchas plantas sometidas a estrés salino se 


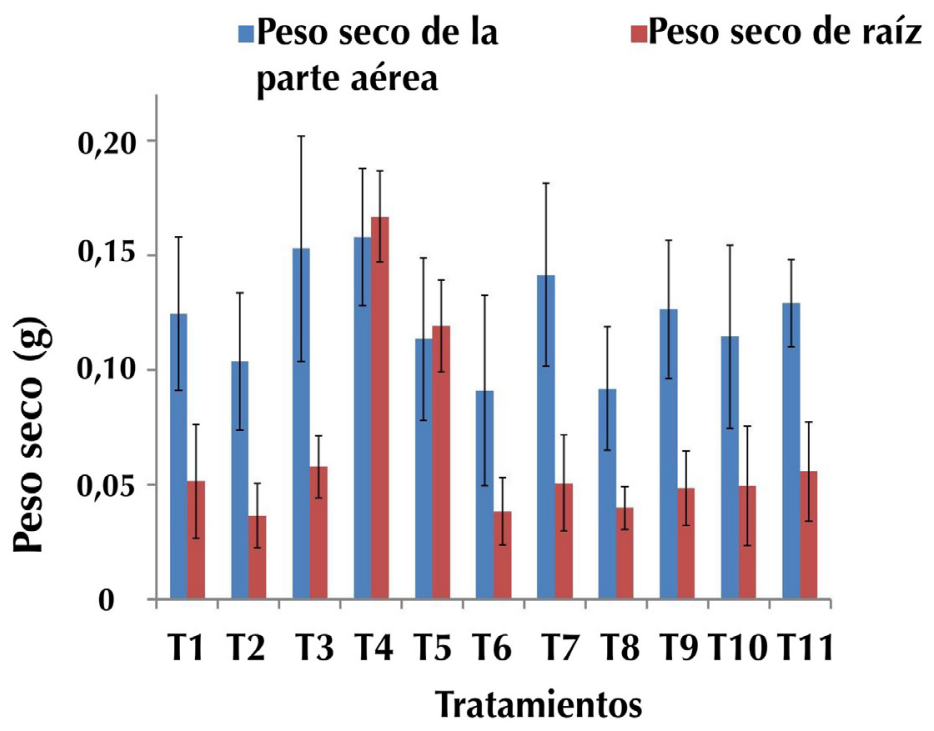

Figura 2. Peso seco parte aérea y raíz de las plantas de Acacia decurrens bajo condiciones de estrés $40 \mathrm{mM} \mathrm{NaCl}$. Cada valor es la media de cinco repeticiones. Las barras de error representan la desviación estándar.

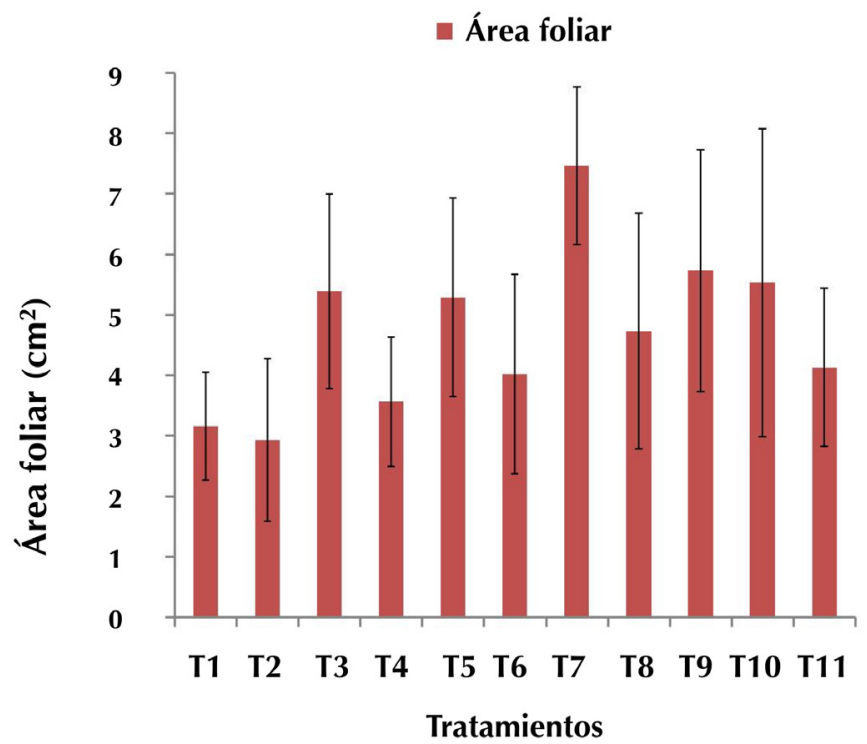

Figura 3. Área foliar de las plantas de Acacia decurrens bajo condiciones de estrés $40 \mathrm{mM} \mathrm{NaCl}$. Cada valor es la media de tres repeticiones. Las barras de error representan la desviación estándar.

asocia muchas veces con una reducción en su actividad fotosintética (Hajlaoui et al. 2009). La fotosíntesis inducida por el estrés salino puede ser relacionados con el cierre de los estomas parcial y factores estomáticos (Das et al. 2008). El aumento en área foliar es indicativo de un incremento en la tasa de actividad fotosintética en las plantas. Esto representa mayores niveles en el contenido de carbono producto de la fijación y en consecuencia un mayor tamaño de la planta (Taiz y Zeiger 2010). El incremento en la actividad fotosintética se puede asociar al efecto benéfico de las bacterias promotoras de crecimiento sobre el crecimiento vegetal bajo condiciones de estrés salino. De forma general a partir de la evaluación de la respuesta vegetal 
de $A$. decurrens a la inoculación con bacterias promotoras de crecimiento bajo estrés salino, es posible establecer que los resultados de esta investigación son pioneros en el área, ya que no se registran estudios previos acerca de la actividad de promoción de crecimiento con bacterias asociadas con A. decurrens, Pantoea deleyi, Enterobacter amnigenus y Serratia liquefaciens son reportadas por primera vez en este estudio por su efecto positivo en el crecimiento vegetal bajo condiciones de estrés salino. Sin embargo su efecto como bacterias promotoras de crecimiento vegetal con otros atributos y actividades ya ha sido reportado. Zahir et al. (2009) encontraron efectos positivos de Serratia sp. en relación al crecimiento y desarrollo de trigo bajo condiciones de estrés salino. Según Bashan et al. (2004), Son et al. (2006) y Selvakumar et al. (2008) las bacterias del género Pantoea son bacterias del suelo promotoras de crecimiento asociadas a un gran número de especies vegetales de importancia agroeconómica. Reis et al. (2000) describen una nueva especie de bacteria endófita del género Pantoea en el cultivo de caña de azúcar, la cual estimula notablemente el crecimiento y raíz de las plantas.

Respecto a las bacterias del género Enterobacter, Morales et al. (2011) evaluaron la capacidad para adherirse a las plantas de maíz y colonizarlas. Veinte días después de la inoculación, reportaron una biomasa mayor de las plantas inoculadas con respecto a las no inoculadas. Yan y Ji (2013) reportaron la capacidad de promoción de crecimiento de Enterobacter sp. R4-368 en plantas de Jatropha curcas inoculadas, evidenciándose un incremento medio del 25,3\%; 77,7\%; 27,5\% y $45,8 \%$ en las variables altura de planta, número de hojas, contenido de clorofila y volumen del tallo, respectivamente. Adicionalmente, la cepa en mención fue capaz de colonizar tejidos en raíz y tallo y aumentar la productividad de las semillas en suelo esterilizado y no esterilizado.

\section{Identificación molecular de aislamientos}

A partir del análisis de los resultados de la respuesta vegetal a estrés salino e inoculación bacteriana, se seleccionaron los tres aislamientos que produjeron una mayor promoción de crecimiento a partir de las variables evaluadas en Acacia decurrens para ser identificados molecularmente. Los aislamientos ACSA12, ACSA14 y ACSA19 fueron identificados como Pantoea deleyi ACSA12, Enterobacter amnigenus ACSA14 y Serratia liquefaciens ACSA19.

\section{CONCLUSIONES}

El desarrollo de la investigación permitió establecer que la inoculación con los aislamientos promotores de crecimiento vegetal Pantoea deleyi ACSA12, Enterobacter amnigenus ACSA14 y Serratia liquefaciens ACSA19 alivianan los efectos bajo condiciones de estrés salino, dando resultados positivos para los parámetros evaluados. De acuerdo con lo anterior, este estudio sirve como base para identificar microorganismos que podrían ser eficientes en la promoción de crecimiento y rendimiento de leguminosas como Acacia decurrens bajo condiciones de estrés salino en campo, fenómeno que se está incrementando en suelos del altiplano Cundiboyacense y que disminuye la productividad de los sistemas ganaderos por salinidad en campo. Se recomienda evaluar los microorganismos con mayor potencial ACSA12 ACSA14 y ACSA19 en 
invernadero durante la duración del ciclo de la Acacia decurrens y la compatibilidad entre las rizobacterias seleccionadas. Adicionalmente determinar la actividad ACC-deaminasa de las cepas que demostraron aliviar el estrés salino de manera significativa en $A$. decurrens.

\section{AGRADECIMIENTOS}

Ministerio de Agricultura y Desarrollo Rural Colombiano.

\section{REFERENCIAS}

Babana, A., Dicko, A., Maïga, K. and Traoré, D. 2013. Characterization of rock phosphate-solubilizing microorganisms isolated from wheat (Triticum aestivum L.) rhizosphere in Mali. Journal of Mıcrobıology and Mıcrobıal Research 1(1):1-6.

Bacilio, M., Rodriguez, H., Moreno, M., Hernandez, J. and Bashan, Y. 2004. Mitigation of salt stress in wheat seedlings by a Azospirillum lipoferum. Biology and Fertility of Soils 40:188-193.

Bashan, Y., Holguin, G. and De-Bashan, L. 2004. Azospirillum plant relationships: physiological, molecular, agricultural, and environmental advances (19972003). Canadian Journal of Microbiology 50(8): p521-577.

Bhattacharyya, P. and Jha, D. 2012. Plant growth-promoting rhizobacteria (PGPR): emergence in agriculture. World Journal of Microbiology and Biotechnology 28(4):1327-1350.
Calvachi, B. 2012. Los ecosistemas semisecos del altiplano cundiboyacense, bioma azonal singular de Colombia, en gran riesgo de desaparición. Mutis 2(2):26-59.

Chen, Y., Rehha, P., Arun, A., Shen, F., Lai, W. and Young, C. 2006. Phosphate solubilizing bacteria from subtropical soil and their Tricalcium phosphatesolubilizing abilities. Applied Soil Ecology 34:33-41.

Costa, E., Nóbrega, R., Carvalho, F., Trochmann, A., Ferreira, L. and Moreira, F. 2013. Plant growth promotion and genetic diversity of bacteria isolated from cowpea nodules. Pesquisa Agropecuária Brasileira 48(9):1275-1284.

Das-Neves, J., Ferreira, L., Vaz, M. and Gazarini, L. 2008. Gas exchange in the salt marsh species Atriplex portulacoides L. and Limoniastrum monopetalum L. in Southern Portugal. Acta Physiologiae Plantarum 30:91-97.

Dubois, M., Gilles, K., Hamilton, J., Rebers, P. and Smith, F. 1956. Colorimetric method for determination of sugars and related substances. Analytical Chemistry 28(3):352-356.

Fiske, C. and Subbaro Y. 1925. The colorimetric determination of phosphorus. Journal of Biological Chemistry 66:375 p.

Ghosh, S., Sengupta, C., Maiti, T. and Basu, P. 2008. Production of 3 -indolylacetic acid in root nodules and culture by a Rhizobium species isolated from 
root nodules of the leguminous pulse Phaseolus mungo. Folia Microbiological 53:351-355.

Glickman, E. and Dessaux, Y. 1995. A Critical examination of the specificity of the salkowsky reagent for indolic compounds produced by phytopathogenic bacteria. Applied and Environmental Microbiology 61(2):793-796.

Goswami, D., Dhandhukia, P., Patel, P. and Thakker, J. 2014. Screening of PGPR from saline desert of Kutch: Growth promotion in Arachis hypogeal Bacillus licheniformis A2. Microbiological Research 169(1):66-75.

Hajlaoui, H., Denden, M. and Bouslama, M. 2006. Effet du chlorure de sodium sur les critèresmorpho-physiologiques et productifs du poischiche (Cicer arietinum L.).]- Annales de Institut National de Recherche en Génie Rural Eaux et Forêts 8:171-187.

Illmer, P. and Schineer, F. 1995. Solubilization of inorganic calcium phosphates solubilization mechanisms. Soil Biology and Biochemistry 27(3):257-263.

Joseph, B., Ranjan, P. and Lawrence, R. 2007. Characterization of plant growth promoting rhizobacteria associated with chickpea (Cicer arietinum L.). International Journal of Plant Production (1):141-152 .

Kang, S., Khan, A., Waqas, M., You, Y., Kim, J., Kim, J., Hamayun, $M$ and
Lee, I. 2014. Plant growth-promoting rizobacteria reduce adverse effects of salinity and osmotic stress by regulating phytohormones and antioxidants in Cucumis sativus. Journal of Plant Interactions 9(1):673-682.

Kavamura, V., Santos, S., Silva, J., Parma, M., Ávila, L., Visconti, A., Zucchi, T., Taketania, R., Andreote, F. and Melo, I. 2013. Screening of Brazilian cacti rhizobacteria for plant growth promotion under drought. Microbiological Research 168(4):183-191.

Lamz, A. y González, M. 2013. La salinidad como problema en la agricultura: la mejora vegetal una solución inmediata. Cultivos Tropicales 34(4):31-42.

Martínez, L. y Navia, J. 2011. Evaluación del comportamiento de algunas especies arbóreas y arbustivas bajo dos distancias de siembra. Revista de Ciencias Agrícolas 28(2):129-36.

Martínez, L. y Navia, J. 2012. Evaluación del comportamiento de algunas especies arbóreas y arbustivas bajo dos distancias de siembra. Ciencias Agrícolas 28(2):129-136.

Mohamed, H. and Gomaa, E. 2012. Effect of plant growth promoting Bacillus subtilis and Pseudomonas fluorescens on growth and pigment composition of radish plants (Raphanus sativus) under $\mathrm{NaCl}$ stress. Photosynthetica 50(2):263-272. 
Morales, Y., Juarez, D., Aragón, C., Mascarua, M., Bustillos, M., Fuentes, L. and Muñoz, J. 2011. Growth response of maize plantlets inoculated with Enterobacter spp., as a model for alternative agriculture. Revista Argentina de Microbiología 43(1):287-293.

Muñoz, D., Calvache, D. y Yela, J. 2013. Especies forestales con potencial agroforestal para las zonas altas en el departamento de Nariño. Revista de Ciencias Agrícolas 30(1):38-53.

Oliveira, C., Alves, V., Marriel, I., Gomes, E., Scotti, M., Carneiro, N., Guimarães R., Shaffert R. and Sá, N. 2009. Phosphate solubilizing microorganisms isolated from rhizosphere of maize cultivated in an oxisol of the Brazilian Cerrado Biome. Soil Biology and Biochemistry 41(9):1782-1787.

Paredes, M. y Espinosa, D. 2010. Ácidos orgánicos producidos por rizobacterias que solubilizan fosfato: una revisión crítica. Terra Latinoamericana 28(1):6170.

Parida, S. and Das, A. 2005. Salt tolerance and salinity effects on plants. Ecotoxicol Environmental Safety. 60: 324-349.

Percival, Y., Himmel, M. and Mielenz, J. 2006. Outlook for cellulase improvement: screening and selection strategies. Biotechnology Advances 24(5):452-481.

Ramírez, M., Piña, Y., Ordoñez, L., Bracho, B. y García, D. 2014. Efecto del $\mathrm{NaCl}$ en plántulas de curarí (Tabebuia serratifolia) en condiciones de laboratorio. Pastos y Forrajes 37(1):17-23.

Rastelli, E., Giraffa, G., Carminati, D., Parolari, G. and Barbuti, S. 2005. Identification and characterisation of halotolerant bacteria in spoiled dry-cured hams. Meat science 70(2):241-246.

Reis, F., Silva, L., Reis, V. e Döbereiner, J. 2000. Ocorrência de bactérias diazotróficas em diferentes genótipos de cana-deaçúcar. Pesquisa Agropecuária Brasileira 35(5):985-994.

Sánchez, D., Gómez, R., Garrido, M. y Bonilla, R. 2012. Inoculación con bacterias promotoras de crecimiento vegetal en tomate bajo condiciones de invernadero. Revista Mexicana de Ciencias Agrícolas 3(7):1401-1415.

Sandhya, V., Ali, S., Venkateswarlu, B., Reddy, G. and Grover, M. 2010. Effect of osmotic stress on plant growth promoting Pseudomonas spp. Archives of Microbiology 192(10):867-876.

Selvakumar, G., Kundu, S., Joshi, P., Nazim, S., Gupta, A., Mishra, P. and Gupta, H. 2008. Characterization of a cold-tolerant plant growth-promoting bacterium Pantoea dispersa 1A isolated from a sub-alpine soil in the North Western Indian Himalayas. World Journal of Microbiology and Biotechnology 24(7):955-960. 
Sindhu, S. and Dadarwal, K. 2001. Chitinolytic and cellulolytic Pseudomonas sp. antagonistic to fungal pathogens enhances nodulation by Mesorhizobium sp. Cicer in chickpea. Microbiological Research 156:353-358.

Son, H., Park, G., Cha, M. and Heo, M. 2006. Solubilization of insoluble inorganic phosphates by a novel salt-and $\mathrm{pH}$ tolerant Pantoea agglomerans R-42 isolated from soybean rhizosphere. Bioresource Technology 97(2):204-210.

Taiz, L. and Zeiger, E. 2010. Plant Physiology. Edition 5\%.(USA): Sinauer Associates, Inc. Publishers, Maryland. USA, p756-770.

Tank, N. and Saraf, M. 2010. Salinity-resistant plant growth promoting rhizobacteria ameliorates sodium chloride stress on tomato plants. Journal of Plant Interactions. 5(1):51-58.

\section{Upadhyay, S., Singh, J. and Singh, D. 2011.} Exopolysaccharide-producing plant growth-promoting rhizobacteria under salinity condition. Pedosphere 21(2):214-222.

Walpola, B. and Yoon, M. 2013. In vitro solubilization of inorganic phosphates by phosphate solubilizing microorganisms. African Journal of Microbiology Research. 7(27):3534-3541.
Wani, P., Khan, M. and Zaidi, A. 2007. Effect of metal tolerant plant growth promoting Bradyrhizobium sp. (vigna) on growth, symbiosis, seed yield and metal uptake by green gram plants. Chemosphere 70:36-45.

Yan, H. and Ji, L. 2013. Improvement of plant growth and seed yield in Jatropha curcas by a novel nitrogen-fixing root associated Enterobacter species. Biotechnology for Biofuels 6:140.

Yasmm, F., Othman, R., Saad, M. and Sijam, K. 2007. Screening for beneficial properties of Rhizobacteria isolated from sweet potato rizosphere. Journal of Biotechnology 6(1):49-52.

Zahir, Z., Ghani, U., Naveed, M., Nadeem, S. and Asghar, H. 2009. Comparative effectiveness of Pseudomonas and Serratia sp. containing ACC-deaminase for improving growth and yield of wheat (Triticum aestivum L.) under-salt stressed conditions. Archives of Microbiology 191:415-424.

Zaidi, A., Khan, M., Ahemad, M. and Oves, M. 2009. Plant growth promotion by phosphate solubilizing bacteria. Acta Microbiological et Immunological Hungarica 56(3):263-284.

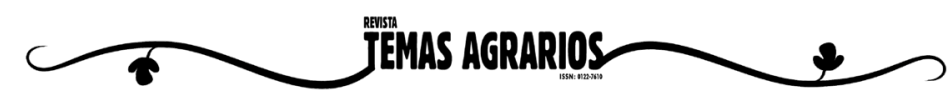

